\title{
NUEVO PERFIL PROFESIONAL EN HOSPITALES: GESTOR DE DOCUMENTACIÓN SANITARIA
}

\section{New professional profile in hospitals: health documentation manager}

Marta Rivas-Gayo, Francisco-Javier Rivas-Flores, Juan Medino-Muñoz, Verónica Alonso-Ferreira y Juan-Antonio Martínez-Comeche
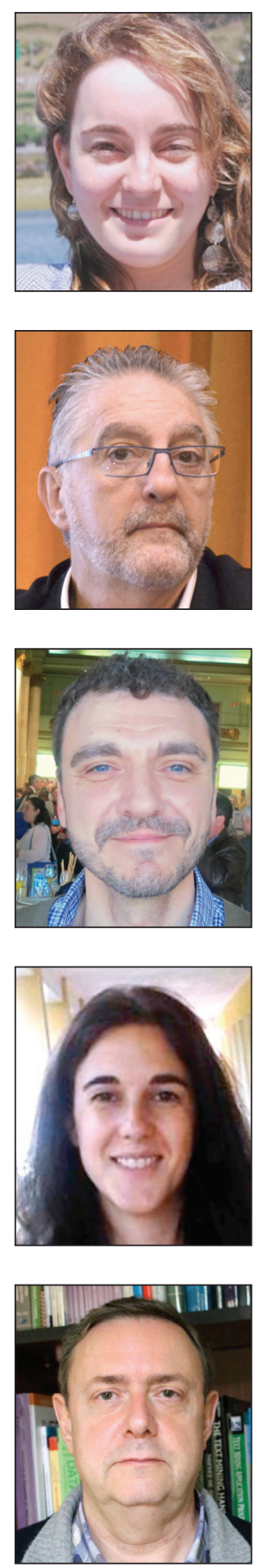

Marta Rivas-Gayo es técnico superior en documentación sanitaria, graduada y diplomada en biblioteconomía y documentación por la Universidad Complutense de Madrid, master universitario en gestión de la documentación, bibliotecas y archivos por la misma universidad y doctoranda en ciencias de la documentación. Ha trabajado como técnico en documentación sanitaria en la Fundación Hospital Alcorcón, Hospital Gral. Univ. Gregorio Marañón, y actualmente en el Hospital Virgen del Mar. http://orcid.org/0000-0002-8397-3953

Hospital Virgen del Mar Honduras, 14. 28016 Madrid, España marta.rivas.gayo@gmail.com

Francisco-Javier Rivas-Flores es doctor en medicina por la Universidad Complutense de Madrid, master en administración y dirección de servicios sanitarios por la Universitat Pompeu Fabra y master en bioética por la Universidad Pontificia de Comillas. Ha sido profesor en la titulación de técnico superior en documentación sanitaria y responsable del Archivo y Documentación Sanitaria del Hospital San Rafael. Actualmente es médico de gestión de pacientes del Hospital Universitario Fuenlabrada. http://orcid.org/0000-0002-5522-7507

Hospital Universitario Fuenlabrada Camino del Molino, 2. 28942 Fuenlabrada (Madrid), España franciscojavier.rivas@salud.madrid.org

Juan Medino-Muñoz es licenciado en geografía e historia por la Universidad Complutense de Madrid y master en información y documentación por la Universidad Carlos III de Madrid. Trabajó como bibliotecario en varias universidades madrileñas y desde 2003 es bibliotecario en el Hospital Universitario de Fuenlabrada.

http://orcid.org/0000-0003-2827-8760

Hospital Universitario Fuenlabrada Camino del Molino, 2. 28942 Fuenlabrada (Madrid), España juan.medino@salud.madrid.org

Verónica Alonso-Ferreira es doctora en biología por la Universidad Complutense de Madrid y científica titular del Instituto de Investigación de Enfermedades Raras (IIER) del Instituto de Salud Carlos III (ISCIII), donde desarrolla su actividad como investigadora en epidemiología y registro de las enfermedades raras.

http://orcid.org/0000-0002-8560-1336

Instituto de Investigación de Enfermedades Raras. Instituto de Salud Carlos III Avda. Monforte de Lemos, 5. 28029 Madrid, España valonso@isciii.es

Juan-Antonio Martínez-Comeche, premio extraordinario de licenciatura y premio extraordinario de doctorado en filología hispánica por la Universidad Autónoma de Madrid, es profesor titular de universidad en la Facultad de Ciencias de la Documentación de la Universidad Complutense de Madrid, especializado en recuperación de información y en comportamiento informacional, temas sobre los que ha dirigido diversas tesis doctorales. Es director del grupo de investigación complutense Griweb, enfocado a la gestión y recuperación de información en la web.

http://orcid.org/0000-0001-9074-8116

Universidad Complutense de Madrid, Facultad de Ciencias de la Documentación Santísima Trinidad, 37. 28010 Madrid, España juan.comeche@pdi.ucm.es 


\title{
Resumen
}

La introducción de la historia clínica electrónica (HCE) en prácticamente todos los centros asistenciales, desplazando y anulando la historia clínica tradicional en papel, ofrece oportunidades extraordinarias de organizar, normalizar y homogeneizar la información sanitaria contenida en las mismas. Los roles profesionales de bibliotecario/a y documentalista hospitalarios convergen en una nueva figura profesional emergente: el gestor de información sanitaria (un profesional que conoce y utiliza las clasificaciones, taxonomías y ontologías con las que se codifican las HCE pero que a la vez integra herramientas bibliográficas en su actividad).

\section{Palabras clave}

Clasificaciones sanitarias; Taxonomías sanitarias; Ontologías sanitarias; Gestores de información sanitaria; Perfiles profesionales; Documentación sanitaria.

\begin{abstract}
The introduction of electronic health records (EHR) in almost all healthcare settings, and the end of traditional paper records, offers new opportunities to organize, normalize, and standardize health information. The professional roles of the librarian in hospitals has converged into a new emerging professional figure: the health documentation manager (a professional who knows and uses health classifications, taxonomies, and ontologies that are encoded within EHRs; but also integrates bibliographic tools into their activities).
\end{abstract}

\section{Keywords}

Health classifications; Health taxonomies; Health ontologies; Health documentation managers; Professional profiles; Health documentation.

Rivas-Gayo, Marta; Rivas-Flores, Francisco-Javier; Medino-Muñoz, Juan; Alonso-Ferreira, Verónica; Martínez-Comeche, Juan-Antonio (2016). "Nuevo perfil profesional en hospitales: gestor de documentación sanitaria”. El profesional de la información, v. 25, n. 2, pp. 279-285.

http://dx.doi.org/10.3145/epi.2016.mar.15

\section{Introducción}

En los últimos años la dinámica asistencial y de investigación ha cambiado de manera notoria en los hospitales y centros sanitarios. La actividad asistencial precisa contar de manera rápida y ágil con los conocimientos más actuales para garantizar la máxima seguridad para el paciente en la atención sanitaria. Para ello no sólo hay que tener localizados los algoritmos de trabajo más recientes y las guías de práctica clínica más completas, sino también poder realizar búsquedas bibliográficas instantáneas según se elabora la historia clínica de los pacientes, que permitan una toma de decisiones más adecuada a las necesidades clínicas del personal sanitario.

Documentalistas y bibliotecarios sanitarios se encuentran en una encrucijada que puede tener importantes repercusiones en su futuro profesional, debido al auge de la tecnología aplicada a las ciencias documentales en cualquiera de sus especialidades. Este campo está siendo copado por especialistas en sistemas de información y en técnicas de computación que aportan indudables ventajas al trabajo documental al dotarlo de herramientas que pueden facilitar, entre otras cuestiones, los mapeos terminológicos o confeccionar ontologías sobre las ciencias de la salud. Ante esta situación surge la pregunta de dónde quedan los profesionales de la información y qué papel están llamados a desempeñar.

El auge de las tecnologías informáticas ha tenido su aplicación más práctica en el desarrollo informático del documento base de la actuación sanitaria profesional que es la historia clínica. La informatización de la historia clínica (figura
1) alcanza en muchos lugares prácticamente al 100\% de la actividad asistencial, e incluso se escala a distintos niveles, como pueden ser el autonómico o el nacional.

La implementación de la Historia Clínica Digital del Sistema Nacional de Salud (HCDSNS) (MSSSI, 2015) permite que la historia clínica de un ciudadano pueda estar disponible en otra comunidad autónoma diferente de donde se generó. Ha sido preciso determinar la tipología documental y otros requisitos de normalización para que pudieran ser accesibles desde las diferentes comunidades. Esta labor, que se plasma en utilidades informáticas, no puede ser llevada a cabo sin el apoyo de expertos documentalistas que lleven el peso de la organización documental.

Las posibilidades que ofrece la informática de enlazar los términos contenidos en la historia clínica (HCE) con lo que se encuentra en la Red, son enormes y permiten al médico acceder casi inmediatamente a la información más actual referente al problema o investigación que está llevando a cabo. El mercado ofrece productos bibliográficos point-ofcare $(P o C)$ que asisten al clínico con la síntesis de la evidencia más reciente y contrastada disponible (Brasure et al., 2014). Aunque la intención en el diseño de estas herramientas PoC es la de ayudar (también en la búsqueda), es evidente que el uso de lenguaje natural no favorece el enlace entre HCE y PoC dado que hay mucho ruido documental debido a sinonimias, topónimos, polisemias, etc., que entorpecen las búsquedas. Esta circunstancia se solventa cuando se cuenta con expresiones normalizadas, generalmente a través de códigos numéricos o alfanuméricos, que identifican de manera unívoca términos literales y códigos. 


\section{Nuevo rol del profesional de la información en el contexto sanitario}

En este trabajo se trata de destacar la importancia de disponer de unos servicios de documentación capaces de dar respuesta a las necesidades expuestas.

A la vista de la situación académica y profesional actual de los bibliotecarios/documentalistas sanitarios observamos que:

- los roles del documentalista sanitario y del bibliotecario hospitalario, tradicionalmente de espaldas entre sí aunque sobradamente reconocidos en la institución (Perrier et al., 2014), deberían adaptarse a esta nueva realidad y dejar de mantenerse inconexos en su actividad diaria (ambos al servicio de los profesionales sanitarios pero sin compartir conocimiento ni herramientas);

- los perfiles formativo-académicos de documentación sanitaria y biblioteconomía/documentación hospitalaria se mantienen inconexos: módulo de técnico superior y/o licenciado/grado en medicina para el ámbito de documentación clínica vs. formación universitaria en información y documentación para el de biblioteca hospitalaria como requisitos básicos;

- la producción científica de los dos ámbitos de conocimiento, prolífica por otro lado, tampoco interactúa, manteniendo espacios diferenciados tanto en publicaciones como en reuniones científicas. A modo de ejemplo valga el ordinal de las Jornadas de Información y Documentación en Ciencias de la Salud Bibliosalud (ya por su 15a edición en 2014), sin una organización/asociación detrás pero con unos profesionales bibliotecarios/documentalistas de alta productividad y actividad; el de las Jornadas Nacionales de Documentación Médica (16a en 2014); y el Congreso Nacional de Documentación Médica (14a edición en 2015), ambos organizados por la Sedom (Sociedad Española de Documentación Médica).

Por tanto se propone:

- Mayor integración de ambos perfiles profesionales en el ámbito laboral, no tanto para una integración en un perfil único profesional (diferentes disciplinas, diferentes áreas de dependencia administrativa, etc.), como para la creación de dinámicas de trabajo en equipo que en un futuro pudieran aunarse en un servicio/departamento único y central (dependiendo de Gerencia) en la estructura hospitalaria.

- Mayor integración en los planes de estudio de las facultades de información y documentación: ni previamente, ni a partir de la implantación del EEES, se ha incluido el tema de la documentación sanitaria

(Ortiz-Repiso; Calzada-Prado; Aportela-Rodríguez, 2013). Tan sólo se ha localizado un master oficial, el de Gestión de la información y el conocimiento en el ámbito de la salud de la Universidad Autónoma de Barcelona, que no es organizado por un departamento de información y documentación, sino por la Facultad de Medicina. Su contenido se centra en sistemas de información sanitaria/hospitalaria pero desde un punto de vista más informático y de gestión que documental;

- Fortalecer lazos entre ambas titulaciones ofreciendo la convalidación de créditos (varía su número en función de la universidad) a los técnicos superiores en documentación sanitaria que se matriculen en el grado en información y documentación y, en sentido contrario, desde los grados universitarios de tema biblioteconómico/documental e informático hacia los estudios profesionales;

- Dar el paso hacia el nuevo perfil del gestor de información sanitaria. Dicho perfil profesional adoptaría en la práctica diaria los criterios y metodología propia de la documentación sanitaria, que utiliza de manera primaria codificaciones y clasificaciones (tabla 1) basadas en las enfermedades, sus manifestaciones, taxonomía, así como los procedimientos que se utilizan para el tratamiento y control de las enfermedades, agentes etiológicos, fármacos, condiciones, etc., que permitan definir lo que le está pasando a una persona, qué es lo que tiene y qué se ha hecho para resolver su problema. Todo ello sin descuidar a la vez la integración de los recursos bibliográficos disponibles tradicionalmente en las bibliotecas hospitalarias (Medina-Aguerrebere, 2012).

\section{El gestor de información sanitaria actua- rá como nexo de unión entre los siste- mas de información y los lenguajes nor- malizados en el ámbito sanitario}

Por tanto la labor fundamental del gestor de información sanitaria debe ser la de servir de nexo de unión entre los sistemas de información y los lenguajes normalizados en el ámbito sanitario. El conocimiento de estos documentalistas en tesauros, lenguajes y ontologías revierte en una mayor eficacia en los ajustes documentales. Así lo recoge MaríaAsunción García-Martín destacando la importancia que supone poseer el conjunto de habilidades necesarias para reconocer la información sanitaria:

- identificar las fuentes de esta información y utilizarlas para recuperar la información relevante;

- valorar la calidad de esta información y su aplicabilidad en una situación específica;

- analizar, entender y utilizar la información para tomar la mejor decisión.

Estas competencias en el manejo de la información van más
Tabla 1. Comparativa de los sistemas de codificación

\begin{tabular}{|l|c|c|c|c|c|}
\hline & CIE-9-MC & CIE-10 & Snomed & MeSH & Orphanet (*) \\
\hline Exhaustividad & + & ++ & +++ & ++ & ++ \\
\hline Precisión & + & ++ & +++ & +++ & ++ \\
\hline Polisemia & + & +++ & +++ & +++ & ++ \\
\hline Sinonimia & + & +++ & +++ & +++ & ++ \\
\hline Epónimos & + & +++ & +++ & + & ++ \\
\hline
\end{tabular}

* Sólo se centra en enfermedades raras 
allá de las meras habilidades instrumentales para acceder a la misma, ya que plantean reconocer la información sanitaria, identificarla y utilizar las fuentes de información para recuperar la más relevante, valorar su calidad y su aplicabilidad en una situación específica (García-Martín, 2011).

\section{Herramientas a manejar}

El perfil propuesto supone una mayor implicación en el proceso de

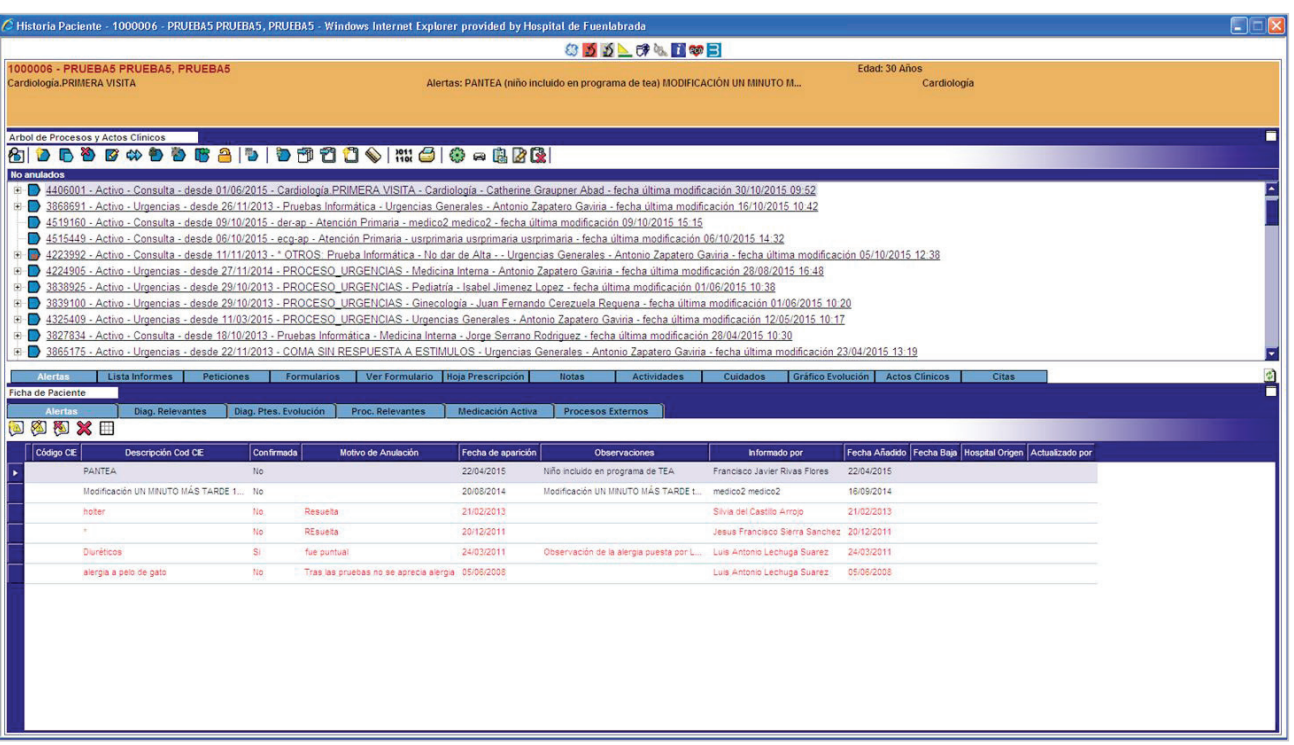

Figura 1. Historia clínica informatizada medicina basada en la evidencia, tanto en su dimensión de búsqueda de las mejores pruebas disponibles sobre determinada cuestión como en su aplicación práctica en la HCE. Es decir, esta nueva dimensión profesional conlleva utilizar, además de las herramientas tradicionalmente utilizadas en los centros de información sanitarios (NLM Classification, Medical Subject Headings MeSH, Descriptores en Ciencias de la Salud DeCS, etc.), las propias del trabajo de codificación: clasificaciones, nomenclaturas, ontologías...

Para definir la actividad asistencial se utilizan clasificaciones de enfermedades de ámbito internacional como la Clasificación Internacional de Enfermedades, en sus versiones CIE-9 (OMS, 2014) y CIE-10 (OMS, 2015). Ambas utilizan criterios taxonómicos, sobre todo en la parte de diagnósticos, organizándose en capítulos según órganos y sistemas, tipo de enfermedades (infecciosas, neoplásicas, etc.) o agentes causales (en el caso de las neoplasias se extiende a identificar la morfología anatomopatológica), y procedimientos que se realizan en los centros sanitarios (ordenados de manera similar a los diagnósticos).

La CIE-10, en pleno proceso de implantación en los hospitales de nuestro país, es mucho más extensa que la CIE-9 ya que llega a niveles de clasificación más precisos. La empresa 3M Health Information Systems, dedicada a la elaboración de herramientas para facilitar la codificación, está llevando a cabo experiencias en este sentido. En concreto está trabajando en sistemas que permitan la traducción de términos clínicos en lenguaje libre a códigos CIE normalizados, para lo que precisa el concurso de técnicos documentalistas que avalen la fiabilidad de las correspondencias (3M, 2014).

Estas clasificaciones tienen sus limitaciones, fundamentalmente en el número de términos que recogen y la rígida estructura que presentan, lo que lleva a la necesidad de contar con otras herramientas con más poder semántico que puedan establecer relaciones entre distintos términos de manera jerárquica.

Por otro lado el proceso de normalización no se limita sólo a la tipología documental sino que debe contar con

herramientas lingüísticas y de uso de terminologías, que permitan en un futuro hacer de estas historias clínicas verdaderos instrumentos activos de trabajo. El objetivo es que faciliten la toma de decisiones, para lo que disponen de algoritmos que de una manera ágil permiten interrelacionar contenidos que pongan a disposición de los médicos los elementos adecuados para tal fin. A esto responde Snomed-CT (Systematized Nomenclature of Medicine-Clinical Terms), la terminología clínica integral, multilingüe y codificada de mayor amplitud, precisión e importancia desarrollada en el mundo. Este producto puede usarse también para codificar, recuperar, comunicar y analizar datos clínicos, permitiendo a los profesionales de la salud representar la información de forma adecuada, precisa e inequívoca. La terminología se constituye de forma básica por conceptos, descripciones y relaciones. Snomed se comporta simultáneamente como:

- terminología de interfase: orientada al ingreso o registro de datos en una HCE, utiliza los términos preferidos por el usuario, intenta ser intuitiva y eficiente para la recolección de datos clínicos;

- terminología de referencia: engloba la colección de conceptos y relaciones usada como punto de referencia común para comparar datos recolectados por múltiples individuos, utilizando variedad de sistemas diferentes en diversas instituciones y viene orientada al análisis de datos.

Por estos motivos Snomed-CT se está convirtiendo en un referente para mejorar la información que pueden ofrecer tanto la HCE como las búsquedas bibliográficas (Bentes-Pinto; De-Oliveira-Rabelo; Torres-Girão, 2014; Carrero-García et al., 2007). De igual modo, permite realizar una metacodificación para la traducción de términos libres en términos codificados y normalizados, lo que puede ser de gran utilidad para búsquedas de información en la Web.

Estos elementos tienen como fin representar con precisión información y conocimiento clínico en el ámbito de la asistencia sanitaria. Se deduce por tanto la importancia de contar con expertos en documentación sanitaria que conozcan 


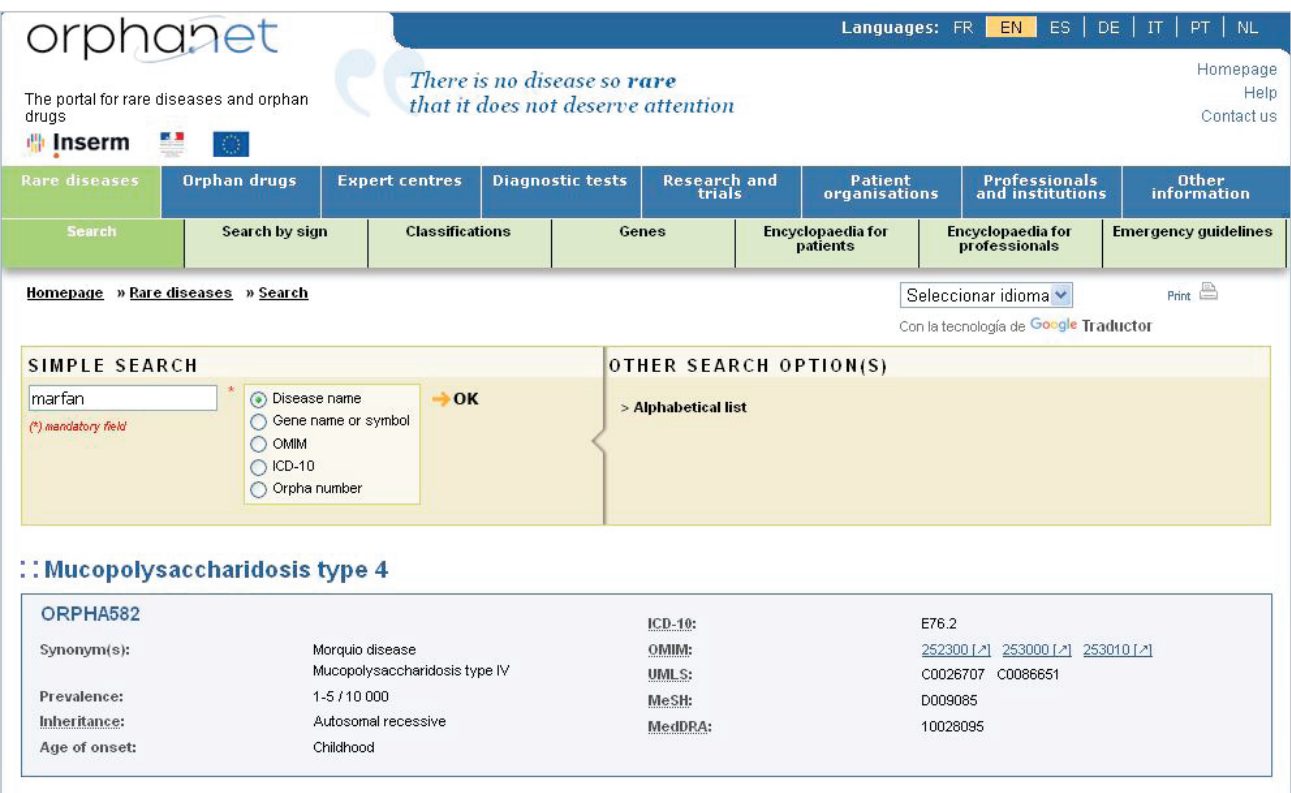

Figura 2. Orphanet, http://www.orpha.net

las herramientas documentales propias, y los lenguajes médicos y sus codificaciones.

Hay que considerar la importancia que puede adquirir el que en los recursos bibliográficos médicos se puedan utilizar para indexar los códigos Snomed u otras nomenclaturas que hagan referencia al problema de salud o a sus manifestaciones, etiología, etc., en lugar de, o además de, las tradicionales palabras clave y/o descriptores. De esta manera se evitaría toda ambigüedad: por ejemplo, si el facultativo quiere incluir el término "acidosis respiratoria" en la HCE (lenguaje natural), el sistema automáticamente lo mapeará a un código normalizado (i.e. E87.2 en CIE-10 o 12326000 en Snomed) que debería poder localizar en una herramienta bibliográfica (base de datos, recurso PoC, etc.) información sobre ese tema de manera unívoca a partir del código introducido.

Una ventaja de la indexación basada en código Snomed es la de favorecer búsquedas más exactas, disminuyendo el ruido documental

Así se ha actuado en el nuevo diccionario (RANM, 2011) de la Real Academia Nacional de Medicina de España (RANM) que, para cada término introducido, incluye tanto el código de las clasificaciones CIE (CIE-9 y/o CIE-10) como los códigos Snomed del mismo. En la actualidad la RANM lidera el proyecto de un diccionario panhispánico de términos médicos (Gallardo-Ponce, 2015). Una de las ventajas que puede ofrecer esta indexación basada en código Snomed es la de favorecer búsquedas más exactas, disminuyendo el ruido documental. Un caso significativo son las búsquedas de enfermedades raras, que por su baja prevalencia e incidencia, precisan ser acotadas lo más posible. El portal Orphanet, específico de enfermedades raras, ya ofrece los códigos de las distintas clasificaciones (CIE-9; CIE-10; Snomed-CT) (figura 2), si bien es preciso mejorar el mapeo (Rivas-Gayo et al.,
2015).

En relación con la historia clínica, la codificación mediante herramientas de mapeo permite recuperar los términos desde texto libre a términos codificados. Actúa de manera no visible para el usuario pero útil para la recuperación de referencias bibliográficas si los términos están indexados, lo que permite disponer de las evidencias más actuales al facilitar búsquedas instantáneas durante la consulta médica (González-Cocina; Pérez-Torres, 2007). En la implementación de estas herramientas están trabajando empresas como:

- Ebsco en su plataforma DynamedPlus (que incluye CIE10);

- Elsevier en su plataforma ClinicalKey (con la taxonomía de elaboración propia EMMeT, Elsevier Merged Medical Taxonomy);

- WoltersKluwer en su archiconocido UpToDate.

Todas ellas con planteamientos en diferentes estados de evolución para facilitar el mapeo entre lenguajes documentales e información clínica.

Otras áreas de actividad en las que se está trabajando son la elaboración de ontologías para un determinado dominio de conocimiento que faciliten la inteligibilidad de lo expresado en estas historias para su difusión entre los usuarios (Sánchez; Batet, 2012).

Las ontologías se utilizan en informática para definir vocabularios que las máquinas puedan entender y sean especificados con la suficiente precisión como para permitir diferenciar términos y referenciarlos de manera precisa. Se entiende por ontologías, en este contexto, la especificación explícita y formal de una conceptualización compartida (Studer; Benjamins; Fensel, 1998). Esta definición supone entender:

- conceptualización: como el modelo abstracto de un fenómeno del mundo del que se han identificado los conceptos relevantes que lo caracterizan;

- su carácter explícito supone que los conceptos utilizados, y las restricciones entre ellos, son definidos de forma declarativa;

- su formalidad implica que es entendible por parte de una máquina y excluye al lenguaje natural;

- ser compartido implica que captura conocimiento consensuado, afecta y es aceptada por un grupo.

De esta manera se puede ir elaborando un corpus de conocimiento que sea utilizado por humanos y máquinas. La inclusión de su uso en las herramientas bibliográficas tradicionales (bases de datos documentales, recursos PoC, bus- 
cadores especializados, etc.) permitiría también dotar de "inteligencia" a los sistemas para que las evidencias con las que trabajan los sanitarios (profesionales e investigadores) sean de más fácil aplicación en el día a día. Iniciativas como PubMed Health o Trip Database trabajan en esta línea de integración de la evidencia.

En la actualidad existen muchas ontologías (Marrero et al., 2010) sobre las que se está trabajando, aunque seguramente sea Galen la más reconocida. Su finalidad es la representación formal de terminologías médicas para los distintos usos clínicos y de codificación. Se implementó para apoyar la elaboración de sistemas clínicos que podrían representar y manipular la información clínica sobre los pacientes en un nivel de detalle suficiente, para apoyar la rutina del cuidado clínico cotidiano (médico y de enfermería) al separar el modelo conceptual del modelo de uso o del idioma. Su utilidad principal es la de representar conceptos complejos y, posteriormente generar clasificaciones automáticas según la perspectiva de interés. Existen experiencias internacionales donde Galen facilitó compartir y mantener el conocimiento médico de una forma consistente.

Los nuevos gestores de información sanitaria tienen la oportunidad de aportar su bagaje de conocimientos en la elaboración de ontologías

\section{Conclusión}

En esta elaboración de dinámicas de trabajo con taxonomías y/o ontologías es necesaria la presencia de documentalistas cuya misión sería dar soporte con los requisitos terminológicos y de relación que se precisan.

La evolución de los sistemas de información a nivel general y en particular en el ámbito sanitario lleva a desarrollar roles diferentes a documentalistas y bibliotecarios hospitalarios, para poder seguir ofertando un servicio de utilidad a la comunidad sanitaria. Su evolución hacia facilitar elementos de búsqueda documental que vaya más allá de las palabras clave o de los tesauros debe ser el camino. Tienen a su favor que cuentan con los conocimientos documentales precisos por su formación, tanto en la atribución de metadatos como en su uso en la búsqueda documental mediante descriptores (controlan la aplicación de los tesauros en ciencias de la salud). Pero precisan avanzar en el conocimiento de nuevos y especializados lenguajes documentales y clasificaciones para facilitar búsquedas más precisas, seguras y rápidas que redunde en el beneficio directo de los pacientes al contar con las evidencias más actuales en tiempo real.

En la elaboración de ontologías, los nuevos gestores de información sanitaria tienen la oportunidad de aportar su bagaje de conocimientos para facilitar una elaboración más adecuada de las mismas así como una validación de las reglas de mapeo entre sistemas como expertos en documentación.

\section{Bibliografía}

3 M Health Information Systems (2014). "Impacto transición CIE10: innovating the language of health". 3M Health Information Systems.

http://www.svdm.es/svdm/wp-content/uploads/2015/06/ P2.pdf

Bentes-Pinto, Virgínia; De-Oliveira-Rabelo, Camila-Regina; Torres-Girão, Igor-Peixoto (2014). "Snomed-CT as standard language for organization and representation of the information in patient records". Knowledge organization, v. 41, n. 4, pp. 311-318.

http://www.repositorio.ufc.br/bitstream/riufc/9858/1/2014_ art_vbpinto.pdf

Brasure, Michelle; Forte, Mary; Butler, Mary; Kane, Robert L. (2014). "Use of evidence-based practice center comparative effectiveness reviews by clinical point-of-care tools". Methods research report. Rockville (MD): Agency for Healthcare Research and Quality (US).

http://effectivehealthcare.ahrq.gov/ehc/products/585/1903/ point-of-care-140428.pdf

Carrero-García, Francisco; Gómez-Hidalgo, José-María; DeBuenaga-Rodríguez, Manuel; Mata, Jacinto; Maña-López, Manuel (2007). "Acceso a la información bilingüe utilizando ontologías específicas del dominio biomédico". Procesamiento del lenguaje natural, n. 38, pp. 107-117. http://rua.ua.es/dspace/handle/10045/3124

Gallardo-Ponce, Isabel (2015). "La RANM lidera un diccionario panhispánico de términos médicos". Diario médico, 26 de octubre.

http://www.diariomedico.com/2015/10/26/area-profesional/ entorno/la-ranm-lidera-un-diccionario-panhispanico-determinos-medicos

García-Martín, María-Asunción (2011). El papel de los bibliotecarios-documentalistas en la docencia en competencias informacionales en ciencias de la salud. http://goo.gl/krCS9r

González-Cocina, Emilio; Pérez-Torres, Francisco (2007). "La historia clínica electrónica. Revisión y análisis de la actualidad. Diraya: la historia de salud electrónica de Andalucía". Revista española de cardiología, v. 7, Supl. C, pp. 37-46.

http://www.revespcardiol.org/es/la-historia-clinicaelectronica-revision/articulo/13108426/

Marrero, Mónica; Sánchez-Cuadrado, Sonia; Urbano, Julián; Morato, Jorge; Moreiro-González, Jose-Antonio (2010). "Sistemas de recuperación de información adaptados al dominio biomédico". El profesional de la información, v. 19, n. 3, pp. 246-254.

http://recyt.fecyt.es/index.php/EPI/article/view/epi.2010. may.04

Medina-Aguerrebere, Pablo (2012). Fuentes de información médica. Barcelona: Editorial UOC, colección El profesional de la infomación, n. 6. ISBN 9788497885560 
MSSSI. Ministerio de Sanidad, Servicios Sociales e Igualdad (2015). Historia Clínica Digital del Sistema Nacional de Salud. http://www.msssi.gob.es/profesionales/hcdsns/home.htm

OMS. Organización Mundial de la Salud (2014). CIE-9-MC: Clasificación internacional de enfermedades, $9^{a}$ revisión, modificación clínica. [Madrid]: Ministerio de Sanidad.

https://eciemaps.mspsi.es/ecieMaps/browser/index_9_mc.html

OMS. Organización Mundial de la Salud (2015). CIE-10-ES: Clasificación internacional de enfermedades, $10^{a}$ revisión, Modificación Clínica: diagnósticos, versión preliminar 2014. [Madrid]: Ministerio de Sanidad.

https://eciemaps.mspsi.es/ecieMaps/browser/index_10_mc.html

Ortiz-Repiso, Virginia; Calzada-Prado, Javier; Aportela-Rodríguez, Ivett M. (2013). “¿Qué está pasando con los estudios universitarios de biblioteconomía y documentación en España?". El profesional de la información, v. 22, n. 6, pp. 505-514.

http://dx.doi.org/10.3145/epi.2013.nov.02

Perrier, Laure; Farrell, Ann; Ayala, A.-Patricia; Lightfoot, David; Kenny, Tim; Aaronson, Ellen; Allee, Nancy; Brigham, Tara; Connor, Elizabeth; Constantinescu, Teodora; MueIlenbach, Joanne; Epstein, Helen-Ann B.; Weiss, Ardis
(2014). "Effects of librarian-provided services in healthcare settings: a systematic review". Journal of the American Medical Informatics Association, v. 21, pp. 1118-1124 http://dx.doi.org/10.1136/amiajnl-2014-002825

Real Academia Nacional de Medicina (2011). Diccionario de términos médicos. Madrid: Editorial Médica Panamericana. ISBN: 9788498351835

Rivas-Gayo, Marta; Rivas-Flores, Francisco-Javier; Alonso-Ferreira, Verónica; Martínez-Comeche, José-Antonio (2015). "Aplicación de distintos sistemas de codificación en la búsqueda bibliográfica de enfermedades raras". En: XIV Congreso nacional de documentación médica. Granada, 1012 junio de 2015.

Sánchez, David; Batet, Montserrat (2013). "A semantic similarity method based on information content exploiting multiple ontologies". Expert systems with applications, v. 40, n. 4, pp. 1393-1399.

http://dx.doi.org/10.1016/j.eswa.2012.08.049

Studer, Rudi; Benjamins, V. Richard; Fensel, Dieter (1998). "Knowledge engineering: principles and methods". Data \& knowledge engineering, v. 25, n. 1-2, pp. 161-197.

http://dx.doi.org/10.1016/S0169-023X(97)00056-6

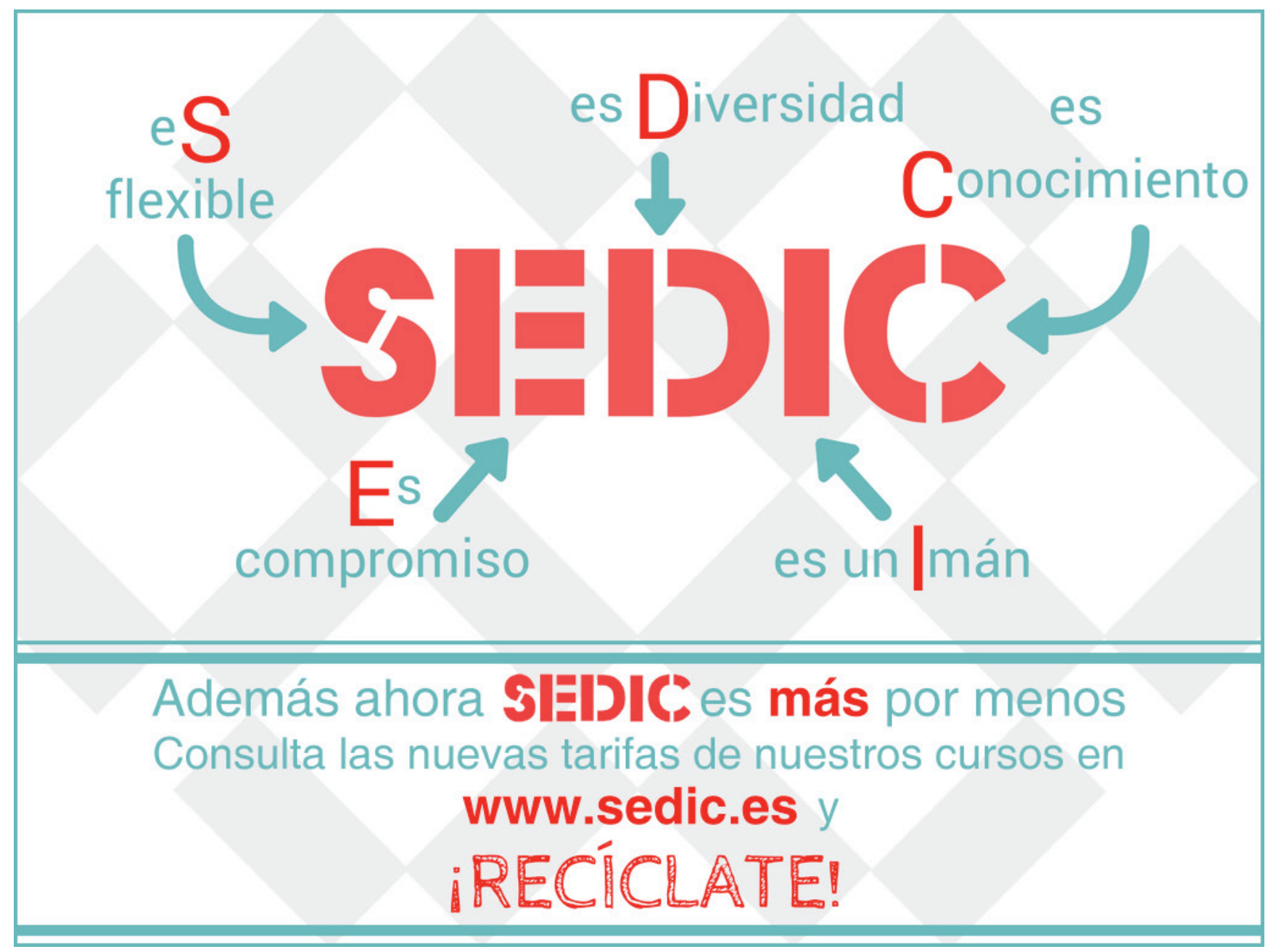

